\title{
Effects of deoxynivalenol- and zearalenone-contaminated feed on the gene expression profiles in the kidneys of piglets
}

\author{
Kondreddy Eswar Reddy ${ }^{1}$, Woong Lee ${ }^{1}$, Jin young Jeong ${ }^{1}$, Yookyung Lee ${ }^{1}$, Hyun-Jeong Lee ${ }^{1}$, \\ Min Seok Kim, Dong-Woon Kim ${ }^{1}$, Dongjo $\mathrm{Yu}^{2}$, Ara $\mathrm{Cho}^{3}$, Young Kyoon $\mathrm{Oh}^{1}$, and Sung Dae Lee ${ }^{1, *}$
}

\begin{abstract}
* Corresponding Author: Sung Dae Lee Tel: +82-63-238-7454, Fax: +82-63-238-7497 E-mail: leesd@korea.kr
\end{abstract}

${ }^{1}$ Animal Nutritional and Physiology Team, National Institute of Animal Science, RDA, Wanju 55365, Korea 2 Swine Science Division, National Institute of Animal Science, RDA, Cheonan 31000, Korea

${ }^{3}$ Animal Disease and Biosecurity Team, National Institute of Animal Science, RDA, Wanju 55365, Korea

ORCID

Kondreddy Eswar Reddy

https://orcid.org/0000-0003-2024-7724

Sung Dae Lee

https://orcid.org/0000-0002-9167-4099

Submitted Jun 13, 2017; Revised Jul 31, 2017; Accepted Aug 31, 2017
Objective: Fusarium mycotoxins deoxynivalenol (DON) and zearalenone (ZEN), common contaminants in the feed of farm animals, cause immune function impairment and organ inflammation. Consequently, the main objective of this study was to elucidate DON and ZEN effects on the mRNA expression of pro-inflammatory cytokines and other immune related genes in the kidneys of piglets.

Methods: Fifteen 6-week-old piglets were randomly assigned to three dietary treatments for 4 weeks: control diet, and diets contaminated with either $8 \mathrm{mg}$ DON$/ \mathrm{kg}$ feed or $0.8 \mathrm{mg}$ ZEN/kg feed. Kidney samples were collected after treatment, and RNA-seq was used to investigate the effects on immune-related genes and gene networks.

Results: A total of 186 differentially expressed genes (DEGs) were screened (120 upregulated and 66 downregulated). Gene ontology analysis revealed that the immune response, and cellular and metabolic processes were significantly controlled by these DEGs. The inflammatory stimulation might be an effect of the following enriched Kyoto encyclopedia of genes and genomes pathway analysis found related to immune and disease responses: cytokine-cytokine receptor interaction, chemokine signaling pathway, toll-like receptor signaling pathway, systemic lupus erythematosus (SLE), tuberculosis, Epstein-Barr virus infection, and chemical carcinogenesis. The effects of DON and ZEN on genome-wide expression were assessed, and it was found that the DEGs associated with inflammatory cytokines (interleukin 10 receptor, beta, chemokine [C-X-C motif] ligand 9, CXCL10, chemokine [C-C motif] ligand 4), proliferation (insulin like growth factor binding protein 4, IgG heavy chain, receptor-type tyrosine-protein phosphatase C, cytochrome P450 1A1, ATP-binding cassette sub-family 8), and other immune response networks (lysozyme, complement component 4 binding protein alpha, oligoadenylate synthetase 2 , signaling lymphocytic activation molecule- 9 , $a$-aminoadipic semialdehyde dehydrogenase, Ig lambda chain c region, pyruvate dehydrogenase kinase, isozyme 4, carboxylesterase 1 ), were suppressed by DON and ZEN.

Conclusion: In summary, our results indicate that high concentrations of DON and ZEN suppress the inflammatory response in kidneys, leading to potential effects on immune homeostasis.

Keywords: Deoxynivalenol; Zearalenone; Pig; Kidney; Gene Expression; Immune System

\section{INTRODUCTION}

Fusarium mycotoxins produced by different fungal species are common contaminants of food and feed ingredients. According to Shephard et al [1], 25\% of the worldwide crop production is contaminated with mycotoxins. Mycotoxin ingestion is considered a severe health problem for both humans and animals. High concentrations of mycotoxins in feed cause major adverse effects; although in a number of cases, mycotoxin levels are too low to produce immediate clinical symptoms. Chronic-level exposure of farm animals to mycotoxins generally results in reduced growth rate, reproductive problems, and increased incidences of different non-infectious and infectious 
diseases, the latter mostly due to repression of the immune function [2] Fusarium mycotoxins are a root cause of substantial economic losses in animal husbandry. Moreover, severe health problems, including severe gastrointestinal tract diseases and malignant tumors, have been reported in both animals and humans, due to long-term exposure to mycotoxins in the diet [3].

Deoxynivalenol (DON) and zearalenone (ZEN) are the most common Fusarium mycotoxins, which are commonly spreading contaminants in animal feed, mostly cereals and forages. These two mycotoxins are characteristically constant under varying environmental conditions and have been identified to cause a diversity of toxic effects in humans, farm animals, and experimental animals. Pigs are more sensitive to DON and ZEN exposure than most other species, partly because of species-specific differences in the metabolism of DON and ZEN [2].

DON can cause toxic effects in a variety of cell systems and various animal species, causing changes in immune cell function, dysregulation of the humoral immune response, and reduced host resistance toward pathogens [4]. DON is also the reason for cytotoxicity to fibroblasts, reticulocytes, and lymphocytes. DON inhibits cell division rate, and RNA and DNA synthesis by binding to ribosomes [5]. At low exposure concentrations, DON upregulates the expression of cytokines and inflammation-related genes, with simultaneous immune stimulation; however, at high exposure, it causes leucocyte apoptosis together with immune suppression [6].

ZEN exhibits strong estrogenic and anabolic activities, causing significant changes in the reproductive system of domestic and laboratory animals [7]. This results from the capacity of ZEN to bind to the estrogen receptors, leading to hyperestrogenism in various animal species, particularly pigs [8]. Once it enters the body, it is primarily metabolized in the liver, which appears to be one of the major targets of this toxin [9]. ZEN also shows many genotoxic and cytotoxic problems in vitro or ex vivo, and is potentially carcinogenic [10]. ZEN is also known to be immunotoxic; however, its function in the inflammatory response is not yet fully understood. ZEN functions as both inductor and suppressor of inflammatory cytokine production [2].

According to the previous research reports of the DON and ZEN effects on pigs; in the present study we are using higher toxic levels of $8 \mathrm{mg} D O N / \mathrm{kg}$ and $0.8 \mathrm{mg} \mathrm{ZEN} / \mathrm{kg}$ feed, respectively, for 4 weeks treatment. To the best of our knowledge, there is no RNA-transcriptome studies were focused on DON and ZEN treated pigs kidney samples. Therefore, in the present study, we perform an RNA-transcriptome analysis for the profiling of gene expression patterns in the kidney samples of control, DON, and ZEN dietary pigs. The RNA-transcriptome analysis would help gain insights into the effects of mycotoxins on the immune- and metabolism-related genes. Identification of the gene expression patterns of various biological processes is significant to understanding the effect of DON and ZEN ingestion. In addition, potential marker genes and affected pathways should be iden- tified and examined to obtain a better understanding of how chronic levels of DON and ZEN-polluted feed affect the kidney function in pigs.

\section{MATERIALS AND METHODS}

\section{Ethics statement}

The protocols for the animal experimental procedures were reviewed and approved by the Institutional Animal Care and Use Committee of the National Institute of Animal Science (No. 2015147).

Animal exposure to DON and ZEN, and the experimental design

The present study was carried out with 15 male castrated 6-weekold male piglets $(\sim 19 \mathrm{~kg})$ purchased from a commercial pig farm. Each piglet was housed in a separate pen $(21 \mathrm{~m} \times 14 \mathrm{~m})$. The piglets were allowed to adjust for 1 week to their new housing at $25^{\circ} \mathrm{C} \pm 1^{\circ} \mathrm{C}$, then assigned to three groups, such as control, DON and ZEN groups (5 piglets/group), with roughly equal total weight per group. The piglets were given a standard diet to meet their nutrient requirements [11], to which, the mycotoxins DON and ZEN were added. Commercially available DON and ZEN powders were properly mixed into the diet at $8 \mathrm{mg} / \mathrm{kg}$ and $0.8 \mathrm{mg} / \mathrm{kg}$, respectively, in the form of purified toxin (Biomin Singapore Pte. Ltd., Singapore). The control feeds, DON- and ZEN- contaminated feeds, and water were provided ad libitum throughout the 4-week experimental period.

\section{Mycotoxin analysis}

DON and ZEN contents were analyzed in DON and ZEN mixed corn feeds by using ultra performance liquid chromatography (UPLC). A homogenized DON mixed grain sample (1 g) was extracted with $20 \mathrm{~mL}$ of distilled water and shake for $30 \mathrm{~min}$, and for ZEN mixed corn sample (1 g), add $0.5 \mathrm{~g}$ of $\mathrm{NaCl}$ and $20 \mathrm{~mL}$ acetonitrile (ACN) then shake for 1 hour. After filtering the extract through a Whatman paper (No. 1), $5 \mathrm{~mL}$ of DON mixed filtrate sample was diluted in $20 \mathrm{~mL}$ of phosphate buffer saline (PBS), and $5 \mathrm{~mL}$ of ZEN mixed grain filtrate sample diluted in $20 \mathrm{~mL}$ of $1 \%$ tween 20 solution. DON and ZEN mixed extract samples were loaded separately onto the appropriate immunoaffinity chromatography columns. The DON mixed sample loaded columns were allowed for completely dried and washed with $10 \mathrm{~mL}$ of PBS and distilled water in the order, and then eluted with $0.5 \mathrm{~mL}$ of $\mathrm{MeOH}$ and $1.5 \mathrm{~mL}$ of ACN. In the case of ZEN, the column washed with $10 \mathrm{~mL}$ of distilled water, then eluted with $1.5 \mathrm{~mL}$ of $\mathrm{MeOH}$. Elutes were dried under $\mathrm{N}_{2}$ gas and dissolved in $1 \mathrm{~mL}$ of $50 \% \mathrm{MeOH}$ then filtered by using a $0.2 \mu \mathrm{m}$ syringe filter, and to be injected into UPLC (Water Acquity UPLC H Class Milford, MA, USA). The photodiode array and fluorescence detector detector were used for DON and ZEN, respectively. The Waters Acquity UPLC BEH C ${ }_{18}$ column $(2.1 \times 100 \mathrm{~mm}, 1.7 \mu \mathrm{m}$ 
particle size) was used for both analyses. We found that, the amounts of DON and ZEN in mixed corn feed were very close to the original concentrations ( $8 \mathrm{mg}$ DON $/ \mathrm{kg}$ feed and $0.8 \mathrm{mg}$ $\mathrm{ZEN} / \mathrm{kg}$ feed). There are no DON and ZEN contaminations were observed in the control feed sample.

\section{Sampling and processing}

After 4 weeks of treatment, all control and DON- and ZEN-treated piglets were killed by an anesthetic overdose, using a combination of barbiturates and pentobarbital. Immediately after the cardiac attest, kidney samples were collected and rapidly frozen in liquid nitrogen, and stored at $-80^{\circ} \mathrm{C}$ for subsequent analysis.

\section{RNA extraction, mRNA library construction, and} sequencing analysis

To construct cDNA libraries from kidney samples, RNA samples were isolated using the TruSeq RNA library kit and purified with Maxwell 16 LEV simply RNA Purification Kit (Promega, Madison, WI, USA), as per the manufacturer's instructions. Initially, the poly-A containing mRNA molecules were purified using poly-T oligo-attached magnetic beads. Post-purification, the mRNA molecules were fragmented into small pieces using divalent cations at ambient temperature. The cleaved RNA fragments were copied into first strand cDNA using reverse transcriptase and random primers. This was followed by second strand cDNA synthesis using DNA polymerase I and RNase $\mathrm{H}$. These cDNA fragments then went through an end repair process, the addition of a single ' $A$ ' base, and finally, the ligation of the adapters. The products were then purified and enriched with polymerase chain reaction to construct the final cDNA library. An RNA-seq library was constructed, and sequencing for each kidney sample was carried out based on protocols for an Illumina HiSeq2000 to generate 90 pair-end reads. The quality of the RNA-seq reads from all kidney samples was checked using FastQC. All the reads passed the quality control checks and were mapped to Sus scrofa from the UCSC using TopHat2 (v2.0.2) and counted using HTseq (v0.5.3p3).

Identification of differentially expressed genes from the control, DON, and ZEN dietary groups

edgeR was used to recognize the differentially expressed genes (DEGs) between the control and DON and ZEN treatment groups [12]. edgeR is designed to analyze replicated count-based expression data and is based on a negative binomial model. Cox-Reid profile methods were used to estimate the dispersion for the pairwise comparisons between control and DON, control and ZEN, and DON and ZEN treatment groups. This was followed by the rest of Cox-Reid common volume dispersion process and statistical analysis, similar to our previous published study [13].

Functional annotation of DON and ZEN differentially expressed genes
The pig Ensembl gene IDs were transformed into official gene symbols by cross-matching with the human Ensembl gene IDs and official gene symbols. The official gene symbols for human homologous of pig DEGs were used for functional clustering and enrichment analysis using the Database for Annotation, Visualization, and Integrated Discovery (DAVID) [14]. The expression of functional groups in DON and ZEN treatments relative to the whole genome was studied using the Expression Analysis Systematic Explorer (EASE) tool within DAVID, which is a modified Fisher's exact test to measure the enrichment of gene ontology (GO) terms. The DON and ZEN treatment DEGs were used to recognize the Kyoto encyclopedia of genes and genomes (KEGG) pathways within DAVID. To identify the enriched GO terms, functionally clustered genes were filtered by an EASE value of 0.01 and preferred.

\section{Statistical analysis}

All sample data are expressed as mean \pm standard error of the mean. Analysis of variance and t-test were carried out to examine the statistical differences between control and mycotoxin dietary groups for all the analyzed parameters. Further variations between means were determined by the least square difference Fisher procedure. Values of $\mathrm{p} \leq 0.05$ were considered significant.

\section{RESULTS}

\section{Identification and analysis of differentially expressed genes}

To study the volume dispersion and the expression profile variations of genes in the pig kidney tissue for each dietary treatment group, DEGs were identified by comparing the expression of the control to DON and ZEN treatments (Supplementary Figure S1). We found a total of 343 DEGs. Out of these, 120 (72 upregulated and 48 downregulated), 66 (35 upregulated and 31 downregulated), and 157 (67 upregulated and 90 down regulated) DEGs were obtained by the comparison of DON and control, ZEN and control, and DON and ZEN, respectively (false discovery rate $<0.01$, fold change $\geq 2$ and $p \leq 0.05$, Figure 1 ). We also illustrated a two-way hierarchical clustering dendrogram for gene expression (log fold change [FC] >2) among DON, ZEN, and the control, which clearly demonstrated that the number of upregulated genes was a little higher than that of downregulated genes in both the dietary treatment groups (Supplementary Figure S2). The expression analysis of DON and ZEN transcripts, comparing each pairwise grouping, is shown in Supplementary Figure S3. The expression level, fold change, p-value, and annotation of DON and ZEN treatment groups for all 23,066 genes identified (data not shown). Owing to the similarity of DON and ZEN Fusarium mycotoxin effects, the RNA-seq data from both DON and ZEN treatment group DEGs were pooled for further analysis.

Gene ontology and the functional annotation of 

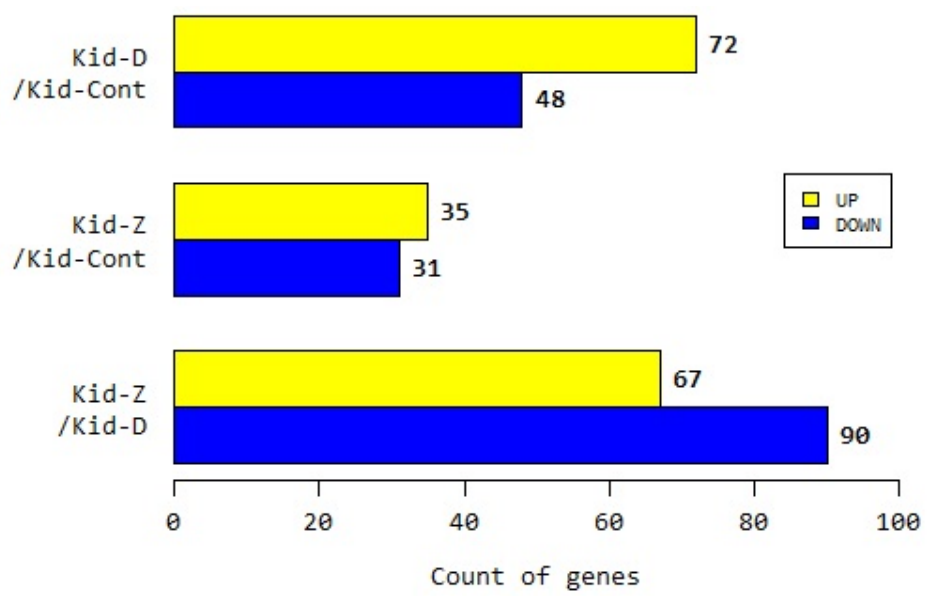

Figure 1. The number of up- and downregulated differentially expressed genes (DEGs) among the control, deoxynivalenol (DON), and zearalenone (ZEN) groups. The Y-axis represents the up- and downregulation of DEGs between control kidneys vs mycotoxin-treatment group kidneys. The $X$-axis represents the total number of transcripts (DEGs for each pairwise comparison were selected at fold change $\geq 2$ and $p \leq 0.05$ ).

\section{differentially expressed genes}

For both DON and ZEN treatment groups, significant DEGs were subjected to GO analysis and compared with the control DEGs. The biological process GO for kidney tissue DEGs of DON- and ZEN-treated pig compared with the control group, are shown in Table 1. The top three biological process GO terms of the DEGs in DON and ZEN were, immune response $(p=0.002)$, chemokine mediated signaling pathway $(\mathrm{p}=0.001)$, and inflammatory response $(\mathrm{p}=0.025)$. The top three molecular function GO terms are chemokine activity $(\mathrm{p}=0.001)$, phosphatidylcholine transporter activity $(p=0.007)$, and heme binding $(p=0.015)$. Finally, the top two cellular components of GO terms were extracellular spaces $\left(\mathrm{p}=4.5 \times 10^{-5}\right)$ and extracellular region $(\mathrm{p}=0.003)$.

\section{Identification of the inflammatory and proliferation-}

related differentially expressed genes

We identified many significant up- and downregulated DEGs, which were inflammatory response-related, immune-related, and disease-associated genes, in both DON and ZEN treatment groups. Some of the significant up- and downregulated genes and their biological processes are listed in Table 2. Some of the important genes related to immune response and proliferation genes, such as cytochrome P450 1A1 (CYP1A1), histone H2B type 1 (HIST1H2BC), kinesin family member 20A (KIF20A), immunoglobulin $\mathrm{G}$ heavy chain $(\mathrm{IgH})$, myomesin-2-like (MYOM2), and stathmin 1 (STMN1), were upregulated. However, the majority of the DEGs associated with immune response, proliferation, and cytokine networks, were suppressed in both DON and ZEN dietary treatment groups, including interleukin 10 receptor beta (IL10RB), chemokine (C-X-C motif) ligand 9 (CXCL9), chemo-

Table 1. Top biological process GO terms of DEGs, obtained from the comparison between the control group and DON- and ZEN-treated groups

\begin{tabular}{|c|c|c|c|c|c|}
\hline Name & GO ID $\mathbf{I D}^{1)}$ & Term $^{2)}$ & Count $^{3)}$ & Genes $^{4)}$ & p-value \\
\hline Biological_process & GO:0006955 & Immune response & 4 & OAS2, CXCL9, CXCL10, CCL4 & 0.002 \\
\hline Biological_process & G0:0065007 & Biological regulation & 6 & CXCL9, IGFBP4, LOC100525546, LOC102158107, C4BPA, PLTP & 0.007 \\
\hline Biological_process & GO:0006954 & Inflammatory response & 3 & $C C L 4, C X C L 9$, CXCL10 & 0.007 \\
\hline Biological_process & GO:0023052 & Signaling & 2 & CXCL9, IGFBP4 & 0.008 \\
\hline Biological_process & GO:0070098 & Chemokine-mediated signaling & 3 & CXCL10, CXCL9, CCL4 & 0.009 \\
\hline Biological_process & GO:0051607 & Defense response to virus & 3 & OAS2, CXCL9, CXCL10 & 0.019 \\
\hline Biological_process & GO:0050789 & Regulation of biological process & 6 & CXCL9, IGFBP4, LOC100525546, LOC102158107, C4BPA, PLTP & 0.016 \\
\hline Biological_process & GO:0043170 & Macromolecule metabolic process & 4 & IGFBP4, LOC100525546, LOC102158107, C4BPA & 0.013 \\
\hline Biological_process & GO:0007154 & Cell communication & 2 & CXCL9, IGFBP4 & 0.005 \\
\hline Biological_process & GO:0006807 & Nitrogen compound metabolic process & 2 & CXCL9, CYP1A1 & 0.012 \\
\hline Biological_process & GO:0007165 & Signal transduction & 2 & CXCL9, IGFBP4 & 0.012 \\
\hline Biological_process & GO:0050794 & Regulation of cellular process & 6 & CXCL9, IGFBP4, LOC100525546, LOC102158107, C4BPA, PLTP & 0.023 \\
\hline Biological_process & GO:0009058 & Biosynthetic process & 2 & PLTP, CYP1A1 & 0.028 \\
\hline Biological_process & GO:0044260 & Cellular macromolecule metabolic process & 4 & IGFBP4, LOC100525546, LOC102158107, C4BPA & 0.028 \\
\hline
\end{tabular}

GO, gene ontology; DEGs, differentially expressed genes; DON, deoxynivalenol, ZEN, zearalenone.

${ }^{1)}$ Gene ontology ID of the biological process. ${ }^{2)}$ Name of the biological term.

${ }^{3)}$ Number of significant genes which are involved in biological terms. ${ }^{4)}$ Name of the genes which are involved in the particular biological term. 
Table 2. Up- and downregulated genes in the kidney samples of DON (8 mg/kg) and ZEN $(0.8 \mathrm{mg} / \mathrm{kg})$ dietary treatment groups of piglets

\begin{tabular}{|c|c|c|c|c|}
\hline Gene name & Description & $\begin{array}{l}\text { Log2 FC } \\
\text { Up/Down }\end{array}$ & $p$-value & Biological process \\
\hline KIF2OA & Kinesin family member $20 \mathrm{~A}$ & +2.11 & 0.045 & Mitotic cytokinesis \\
\hline CYP1A1 & Cytochrome P450 1A1 & +2.01 & 0.043 & Toxin metabolic process \\
\hline $\lg H$ & IgG heavy chain & +8.43 & 0.021 & NF-kappa B signaling pathway \\
\hline BTD & Biotinidase & +2.50 & 0.04 & Nitrogen compound metabolic process \\
\hline$H B M$ & Hemoglobin subunit mu & +2.18 & 0.026 & Extracellular exosome \\
\hline HIST1H2BC & Histone H2B type 1 & +2.10 & 0.012 & Innate immune response \\
\hline CES & Liver carboxylesterase & +6.20 & 0.040 & Carboxylic ester hydrolase activity \\
\hline MYOM2 & Myomesin-2-like & +2.51 & 0.048 & Regulatory mechanisms for expression quantity and temporal appearance \\
\hline STMN1 & Stathmin 1 & +2.23 & 0.049 & Reason for various cancers \\
\hline IL10RB & Interleukin 10 receptor, beta & -2.10 & 0.031 & Cytokine-cytokine receptor interaction \\
\hline CXCL9 & Chemokine (C-X-C motif) ligand 9 & -3.27 & 0.017 & Inflammatory response \\
\hline CXCL10 & Chemokine (C-X-C motif) ligand 10 & -2.37 & 0.029 & Inflammatory response \\
\hline$L Y Z$ & Lysozyme & -2.43 & 0.031 & Lysozyme activity \\
\hline CES1 & Carboxylesterase 1 & -5.61 & 0.000 & Hydrolysis of xenobiotics and drugs \\
\hline$A B C B 8$ & ATP-binding cassette sub-family 8 & -2.27 & 0.032 & Pathologies associated, lipid transport \\
\hline CCL4 & Chemokine (C-C motif) ligand 4 & -2.14 & 0.030 & Inflammatory response \\
\hline ALDH7A1 & $\alpha$-aminoadipic semialdehyde dehydrogenase & -2.03 & 0.025 & Detoxification of aldehydes \\
\hline CABPA & Complement component 4 binding protein a & -2.10 & 0.019 & Response to immune system \\
\hline CYP2B22 & Cytochrome P450 2B22 & -2.38 & 0.034 & Transport, and catabolism, \\
\hline IGFBP4 & Insulin like growth factor binding protein 4 & -2.03 & 0.0001 & Regulation of cell growth \\
\hline MYOM2 & Myomesin-2 & -2.07 & 0.025 & Immunoglobulin subtype \\
\hline PLTP & Phospholipid transfer protein & -2.00 & 0.017 & Lipid metabolic process \\
\hline$S Q R D L$ & Sulfide quinone reductase-like & -2.31 & 0.033 & Oxidoreductase activity \\
\hline THBS4 & Bospondin-4 & -4.11 & 0.0001 & Phagosome activity \\
\hline CYP4X1 & Cytochrome P450 4X1 & -2.51 & 0.049 & Neurovascular function in the brain \\
\hline CYP4A24 & Cytochrome P450 4A24 & -3.20 & 0.029 & catalytic activity of lauric acid and palmitic acid \\
\hline OAS2 & Oligoadenylate synthetase 2 & -2.72 & 0.049 & Immune response \\
\hline PTPRC & Receptor-type tyrosine-protein phosphatase C & -2.09 & 0.030 & Regulate a cell growth, and oncogenic transformation. \\
\hline $\operatorname{lgH}$ & IgG heavy chain & -10.14 & 0.023 & NF-kappa B signaling pathway, \\
\hline IGLC1 & Ig lambda chain c region & -6.15 & 0.027 & Play in diseases such as cancer and diabetes \\
\hline ANGPTL4 & Angiopoietin-like 4 & -2.20 & 0.041 & Negative regulation of endothelial cell apoptotic process \\
\hline PDK4 & Pyruvate dehydrogenase kinase, isozyme 4 & -2.49 & 0.035 & Positive regulation of defense response \\
\hline SLAM9 & Signaling lymphocytic activation molecule-9 & -2.68 & 0.035 & Play a role in the immune response \\
\hline
\end{tabular}

DON, deoxynivalenol; ZEN, zearalenone; FC, fold change.

kine (C-X-C motif) ligand 10 (CXCL10), chemokine (C-C motif) ligand 4 (CCL4), $\alpha$-aminoadipic semialdehyde dehydrogenase (ALDH7A1), complement component 4 binding protein alpha (C4BPA), insulin-like growth factor binding protein 4 , oligoadenylate synthetase 2 (OAS2), receptor-type tyrosine-protein phosphatase $\mathrm{C}(P T P R C)$, IgH, angiopoietin-like 4 (ANGPTL4), pyruvate dehydrogenase kinase, isozyme 4 , and signaling lymphocytic activation molecule- 9 .

\section{KEGG pathway analysis of DON and ZEN dietary group differentially expressed genes}

The GO enrichment analysis was carried out using the DAVID software and knowledgebase to capture the enriched biological terms from the DEGs of both mycotoxin-treated groups. KEGG pathway analysis of DON and ZEN group DEGs showed that the DEGs were mainly clustered into immune response-related, disease-related, metabolism-related, and other pathways. The majority of the pathways obtained from the comparison of DON and ZEN dietary group with the control group were linked with inflammatory and disease pathways (Table 3). The inflammatory response pathways include cytokine-cytokine receptor interaction $(\mathrm{p}=0.015)$, chemokine signaling pathway $(\mathrm{p}=0.044)$, and toll-like receptor signaling pathway $(\mathrm{p}=0.024)$. Some diseaseassociated pathways, such as chemical carcinogenesis $(\mathrm{p}=0.022)$, retinol metabolism $(\mathrm{p}=0.020), \operatorname{SLE}(\mathrm{p}=0.037)$, tuberculosis ( $\mathrm{p}$ $=0.043)$, and Epstein-Barr virus infection $(\mathrm{p}=0.048)$, were also identified. In addition, metabolic pathways $(\mathrm{p}=0.008)$, PI3KAkt signaling pathway $(\mathrm{p}=0.016)$, tryptophan metabolism $(\mathrm{p}$ $=0.015)$, phagosome $(p=0.044)$, and steroid hormone biosynthesis $(p=0.019)$ were identified.

\section{DISCUSSION}

The contamination of animal feed with DON and ZEN is a uni- 
Table 3. Various KEGG pathways observed in the kidney samples of DON- and ZEN-contaminated dietary treatment groups of piglets

\begin{tabular}{|c|c|c|c|c|}
\hline KEGG ID") & KEGG map name ${ }^{2)}$ & Count $^{3)}$ & Gene names ${ }^{4)}$ & $\mathrm{p}$-value \\
\hline 04060 & Cytokine-cytokine receptor interaction & 4 & $C C L 4, C X C L 9, C X C L 10, I L 10 R B$ & 0.015 \\
\hline 01100 & Metabolic pathways & 4 & ALDH7A1, CES1, CYP1A1, CYP2B22 & 0.008 \\
\hline 04062 & Chemokine signaling pathway & 3 & CXCL9, CXCL10, CCL4 & 0.044 \\
\hline 04620 & Toll-like receptor signaling pathway & 3 & $C C L 4, C X C L 9, C X C L 10$ & 0.024 \\
\hline 00380 & Tryptophan metabolism & 3 & ALDH7A1, CYP1A1, CYP2B22 & 0.015 \\
\hline 05152 & Tuberculosis & 2 & IL 10RB, IgH & 0.043 \\
\hline 05169 & Epstein-Barr virus infection & 2 & $\operatorname{IgH}, \operatorname{IL} 10 R B$ & 0.048 \\
\hline 05204 & Chemical carcinogenesis & 2 & CYP1A1, CYP2B22 & 0.022 \\
\hline 05322 & Systemic lupus erythematosus & 2 & HIST1H2BC, IgH & 0.037 \\
\hline 04145 & Phagosome & 2 & $\operatorname{lgH}$, THBS4 & 0.044 \\
\hline 04151 & PI3K-Akt signaling pathway & 2 & THBS4, IgH & 0.016 \\
\hline
\end{tabular}

KEGG, Kyoto encyclopedia of genes and genomes; DON, deoxynivalenol, ZEN, zearalenone.

${ }^{1)}$ KEGG pathway IDs in DON and ZEN treatment groups. ${ }^{2)}$ Name of the KEGG pathway which is identified in DON and ZEN treatment groups.

${ }^{3)}$ Number of significant genes which is involved in KEGG pathways. ${ }^{4)}$ Name of the genes which are involved in the particular KEGG pathways.

versal problem, given the capacity of the fungus that synthesizes DON and ZEN to grow in any environmental condition [2]. DON and ZEN contamination of food and feed has been frequently linked with substantial effects on human and animal health. To date, very little is known about the effects of DON and ZEN on the transcriptional levels in farm animals. Therefore, we used an RNA-seq approach to assess the effects of DON and ZEN on the universal transcriptome expression in the kidneys of pigs fed DON- and ZEN-contaminated diets for 28 days. The analysis and inference of RNA-seq data depends largely on the ultimate goal of the study, which, in this case, was to examine differential gene expression. The RNA-seq data illustrated a wide range of expression levels in the kidney samples, which are stable and reliable in the DON and ZEN dietary treatments. Detection of genes and pathways altered by dietary Fusarium toxins might lead to improved diagnostic, treatment, and prevention strategies for mycotoxicosis.

The RNA-seq data for DON and ZEN showed genes that had an altered profile compared with the control group. Of these genes, 107 were upregulated and 79 were downregulated. Some of the significant up- and downregulated genes and their roles are listed in Table 2. In this study, both mycotoxins were found to affect the immune system, cell proliferation, and cytokine networks. Here, we elucidate some of these significantly affected up- and downregulated genes. However, comparable studies in pigs are not available for each gene, which are needed for further analysis of the obtained results. The RNA-seq screening revealed a significant number of up- and downregulated genes. Increased gene expression was observed in KIF20A (FC: 2.11), CYP1A1 (FC: 2.01), IgH (FC: 8.43), HIST1H2BC (FC: 2.10), and STMN1 (FC: 2.23). KIF20A is a protein-coding gene, which is necessary for the chromosome passenger complex-mediated cytokinesis. The GO annotations for this gene are connected to protein kinase binding and ATPase activity, and it is also associated with immune system pathways and vesicle-mediated transport [15]. The CYP1A1 protein is a monooxygenase; it catalyzes a variety of structurally unrelated compounds such as xenobiotics, and is involved in drug metabolism. This gene is associated with the diseases Ehrlich tumor carcinoma and lung squamous cell carcinoma [16]. IgH is the large polypeptide subunit of an antibody. It recognizes foreign antigens and initiates immune responses such as phagocytosis and induces the complement system. IgH is linked with follicular lymphoma 1 and mantle cell lymphoma, in addition to the immune response mediated by DAP12 receptors in NK cells and immune response Fc epsilon RI [17]. Similar to our results, Butler and Wertz [18] also found that IgH gene expression in piglets is related to the immune response toward various stress conditions. HIST1H2BC is a protein-coding gene, which plays a major role in antifungal, antibacterial, and antimicrobial activities. Tollin et al [19] found that this gene participates in human colonic defense against microbes and fungi. The STMN1 gene is a phosphoprotein that regulates several important cellular functions. The increased expression of STMN1 has been observed in many human cancers and confers a poor prediction. According to Cheng et al [20], the upregulation of STMN1 is seen in primary nasopharyngeal carcinoma, and its expression has been linked with the recurrence and advanced stages of the disease. Many groups have demonstrated that the inhibition of this gene might partly reverse the malignant phenotype.

Using the RNA-seq data, we also recognized some of the significantly downregulated genes associated with inflammatory response, cell proliferation, and cytokine networks. The expression of IL1ORB (FC: -2.10) was downregulated. It is an important anti-inflammatory marker of cytokine signaling gene and is encoded by the cytokine receptor family. It is an accessory chain, necessary for the active interleukin 10 receptor complex. Coexpression of $I L 10 R B$ gene has been shown to be essential for IL10-induced signal transduction. Diseases linked with IL10RB comprise inflammatory bowel disease, and autosomal recessive and early-onset colitis. ILIORB is associated with the immune system pathways and Jak/STAT signaling pathway [21]. Similar to our results, a significant downregulation of IL10 was observed 
in the liver samples of ZEN-fed pigs [22]. Downregulated CXCL9 and CXCL10 genes (FC: -3.27 and FC: -2.37 , respectively) encode small cytokines related to the CXC chemokine family. These two genes have many functions, including chemoattraction for monocytes, NK cells, and T cells, promotion of T cell adhesion to endothelial cells, antitumor activity, and inhibition of bone marrow colony formation and angiogenesis [23]. CXCL9 and CXCL10 are associated with pathways involving $\mathrm{G}$ protein-coupled receptor and peptide ligand-binding receptors. According to Altara et al [24], CXCL9 and CXCL10 are suitable biomarkers for the development of heart failure and left ventricular dysfunction in humans. CCL4 (FC: -2.14) is also a chemokine protein, which is a chemoattractant for natural killer cells, monocytes, and a variety of other immune cells; it gets secreted and has chemokinetic and inflammatory functions. CCL4 is associated with human immunodeficiency virus 1 and meningitis; it is linked with the immune system and toll-like receptor signaling pathways. Johannes et al [25] reported that CCL4 modulates cell death and survival and immunological and hematological diseases, in various animals fed DON- and ZEN-contaminated feed. In the present study, we found that the lysozyme ( $L Y Z$ ) enzyme was significantly downregulated (FC: -2.43); it is an antimicrobial enzyme produced by animals, and is found in the spleen, kidney, plasma, and other tissues. LYZ is mainly involved in the innate immune system, and is associated with amyloidosis and familial visceral diseases. It is related to the immune system and salivary secretion pathways.

Insulin like growth factor binding protein 4 (IGFBP4; FC: $-2.03)$ is a unique gene, which frequently inhibits numerous cancer cells in vivo and in vitro. Its inhibitory action has been exhibited in vivo in prostate and colon cancer. IGFBP4 protein is secreted by most of the colon cancer cells. This gene is linked with the diseases involving insulin-like growth factor and renal osteodystrophy, and is associated with the pathways of $\beta$-catenin-dependent Wnt signaling and myometrial relaxation. According to Shen and Singh [26], IGFBP4 gene expression plays a significant function in the transition from proliferation to separation of a human colon cancer cell line, $\mathrm{CaCo}_{2}$. Wan et al [27] revealed that due to trichothecene toxicity, IGFBP4, an insulin-like growth factor (IGF)binding protein, was suppressed in the rat pituitary adenoma gretchen hagen $3(\mathrm{GH} 3)$ cells, demonstrating that the T-2 toxin gave rise to a growth hormone-IGFBP4 deficiency in the cells. In the present study, the C4BPA gene (FC: -2.10$)$ was downregulated. It is involved in the complement system, acting as an inhibitor. C4BPA binds to apoptotic and necrotic cells, and DNA, facilitating clean up after an injury. A number of fungal and bacterial pathogens bind to the human $C 4 B P A$, which permits them to establish infection. C4BPA is associated with protein S deficiency and acute post-streptococcal glomerulonephritis, and is involved in the immune system and pertussis pathways [28]. PTPRC (FC: -2.09 ) belongs to the protein tyrosine phosphatase $(P T P)$ family. It is a signaling molecule that regulates different cellular processes such as cell growth, mitotic cycle, differentiation, and oncogenic transformation. This gene is an essential regulator of T- and B-cell antigen receptor signaling. It suppresses JAK kinases, and therefore, functions as a regulator of cytokine receptor signaling. Alternatively spliced transcript variants of PTPRC, which encode distinct isoforms, have been reported. This gene is linked with severe combined immunodeficiency, B-cell/ natural killer cell, $\mathrm{T}$ cell-negative and positive selection, and hepatitis $\mathrm{C}$ virus [29]. The most significant immune response gene $\operatorname{IgH}$ (FC: -10.09) was highly downregulated. It is associated with antigen receptors expressed by $\mathrm{B}$ cells and secreted by plasma cells, and is one of the major components of the adaptive immune response. IgH also acts as a cell-surface receptor for antigens, which permits cell signaling and cell activation, and for soluble effector molecules, which can individually bind and neutralize antigens at a distance. The IgA, IgM, and IgG concentrations were significantly decreased in the peripheral blood lymphocytes of the piglets that were fed contaminated feed with various Fusarium mycotoxins [30]. Based on these results, we hypothesized that, in our present study, the toxic levels of DON and ZEN might affect the immune system, and we found that IgH was highly suppressed. The OAS2 gene (FC: -2.72 ) is a member of the $2-5 \mathrm{~A}$ synthetase family, and this gene is mainly involved in the innate immune response to viral infection. OAS2 might also play a role in other cellular processes such as cell growth, differentiation, apoptosis, and gene regulation. It is also associated with the immune system and interferon gamma signaling pathways. Similar to our results, OAS2 gene expression was also suppressed in the lung tissue of mice orally gavaged with DON [31]. In the present study, the ANGPTL4 gene (FC: -2.20$)$ showed downregulation. This gene is associated with hypoxia-induced expression in endothelial cells. It can control angiogenesis and modulate tumorigenesis. ANGPTL4 reduces proliferation, migration, and tubule development of endothelial cells, and decreases vascular leakage. It might also serve a protective function in endothelial cells through endocrine action. It is directly involved in regulating glucose homeostasis, insulin sensitivity, and lipid metabolism, and is associated with severe non-proliferative diabetic retinopathy [32]. Downregulation of ANGPTL4 has also been associated with type 2 diabetes.

Apart from the above-mentioned genes, most of the DEGs mentioned in Table 2. are also associated with the immune response, and inflammatory and proliferation networks. We predict that higher toxic levels of DON and ZEN lead to significant decreases in the gene expression of inflammatory cytokines, and are also connected with a decrease in the level of other inflammatory mediator genes. The expression profiles of DEGs in DON and ZEN treatments showed that most genes were downregulated than upregulated. Due to acute toxicity levels of DON and ZEN, a number of inflammatory and proliferation-associated genes were downregulated, and it was observed that some of the upregulated genes were associated with various diseases. The func- 
tional annotation analysis also illustrated more GO biological process terms and KEGG pathways related to immune system, metabolic processes, and diseases in both DON and ZEN treatment groups (Tables 1, 2, 3).

In KEGG enrichment analysis, we identified significant immune- and disease-associated pathways (Table 3, Supplementary Figure S4). We found some pathways associated with human diseases, and concluded that there might be consequences of DON and ZEN toxicity in humans. Remarkably, almost all genes found in the present study were downregulated. We hypothesized that severe toxic levels of DON and ZEN could modulate and downregulate various genes that inhibit the activation, apoptosis of immune cells. In this study, the most important inflammatory response-related cytokine-cytokine receptor interaction pathway was identified and chemokine genes CCL4, CXCL9, and CXCL10, and the anti-inflammatory gene IL1ORB were found to be downregulated. Cytokines and their specific receptors represent a system that plays a major role in blood and immune cells. Cytokines are vital intercellular regulators and mobilizers of cells engaged in innate as well as adaptive inflammatory host defenses, cell growth, cell death, differentiation, angiogenesis, and development and repair procedures aimed at restoration of homeostasis. According to Amuzie et al [33], DON induces the expression of suppressors of cytokine signaling in mice, and has the potential to play a regulatory role in signaling pathways mediated by the cytokine receptor superfamily members such as $G H$ and inter leukin-6 (IL-6) that eventually affect inflammation and growth, respectively. Suppressor of cytokine signaling -induced negative regulation of cytokine and growth factor pathways has been confirmed in several in vivo and in vitro studies involving a number of species [34]. Metabolic pathways might be involved in different diseases; in this study, metabolic pathways were associated with ALDH7A1, carboxylesterase 1, CYP1A1, and CYP2B22, all of which were downregulated. These genes are associated with various diseases and immune inhibition. The metabolism of most mycotoxins in humans and animals remains unclear. Observed metabolic pathways of trichothecenes, including DON, ZEN, T-2 toxin, 3-aDON, nivalenol, diacetoxy scirpenol, and fusarenon- $X$ in humans, swine, poultry, and ruminants [35]. Toll-like receptor (TLR) signaling pathways regulate the downregulation of CCL4, CXCL9, and CXCL10 genes. TLR signaling pathways can play a major role in the activation of the adaptive immune system by inducing proinflammatory cytokines and upregulating costimulatory molecules of antigen-presenting cells. Activating the TLRs leads to the sensitive expression of different inflammatory genes, which have a protective role against infection. TLRs in renal cells are implicated in innate immune responses in kidney infection. According to Gu et al [36], TLR stimulates DON-exposed intestinal porcine epithelial cells, which could alleviate the reduction in cell survival and proliferation of immune cells.

In the present study, the important inflammatory immune response of chemokine signaling pathway was observed to be significantly expressed. This is a part of the normal immune system function, as chemokines are important components of basal leukocyte trafficking necessary for immune system functioning and development, and immune surveillance. Chemokines also contribute to the growth, differentiation, and activation of leukocytes, and motivates diverse effector functions. Similar to our results, previous studies have reported that the chemokine genes were downregulated in the spleen and intestinal samples of ZENand DON-treated piglets, respectively [37]. In this study, the chemokine genes CXCL9, CXCL10, and CCL4 might be suppressed due to severely toxic levels of DON and ZEN.

We also found some significant disease-related pathways in the present study. However, the role of DON and ZEN on these pathways is not yet fully known. The SLE pathway involves HISTIH2BC and IgH. It is a prototypic autoimmune disease characterized by the creation of IgG autoantibodies and it is particular for self-antigens. Hueza et al [38] showed that ZEN-treated animals showed autoimmune disorders such as SLE. We also found the chemical carcinogenesis pathway, and it is expected that exposure to environmental chemical carcinogens might be responsible for a majority of human and animal cancers. Genotoxic carcinogens such as DON and ZEN can attack biological macromolecules such as RNA and DNA, directly or indirectly, during metabolism, resulting in the formation of adducts with these macromolecules [39]. We also found tuberculosis and Epstein-Barr virus infection pathways, both of which are related to ILIORB and IgH. The immune response of $I L 10 R B$ and $I g H$ genes was downregulated. IgH and THBS4, associated with the phagosome and phosphatidylinositol 3-kinase-Akt (PI3K-Akt) signaling pathways, were also found to be downregulated. Phagocytosis is the process of taking in comparatively large particles by a cell, and is an essential mechanism in tissue remodeling, inflammation, and protection against infectious agents. According to Food Research Institute, DON inhibits phagocytosis, and consequently, causes immunosuppressive effects. PI3K-Akt signaling pathway is stimulated by several types of cellular stimuli or toxic exposures, and controls primary cellular roles, including transcription, translation, growth, and proliferation. It is mainly involved in a broad range of biological processes, such as apoptosis and intestinal inflammation, and the intestinal barrier. Zhang et al [40] revealed that PI3K/Akt pathway was altered due to DON-mediated intestinal toxicity in porcine epithelial cells exposed to $20 \mu \mathrm{M}$ DON for $60 \mathrm{~min}$.

A number of studies suggest that DON and ZEN are powerful immune modulators, acting as immunosuppressive as well as immune-stimulatory agents [6]. This is strongly supported in our present study. For instance, as discussed earlier, most of the immune-related DEGs and pathway associated gene expression profiles were altered (suppressed or upregulated) due to severely toxic levels of DON and ZEN. A majority of in vivo studies have focused on a broad array of pro-inflammatory cytokines and chemokines that are noticeable in various organs such as liver, spleen, kidney, and lung, after DON and ZEN exposure [37]. 
The kidney might play the primary role in the inflammatory response along with the growth of acquired immunity. DON and ZEN stimulate apoptosis, dysfunction, inflammatory response, and oxidative stress in mouse kidney [41]; however, very little data are available on the in vivo effects of DON and ZEN on the modulation of kidney inflammatory markers in pigs. Our study demonstrated that a number of significantly affected genes following the exposure to DON- and ZEN-contaminated diet treatments were associated with inflammatory response. Our inflammatory gene expression profile is somewhat similar to those identified using microarray analysis in spleen samples of ZEN-fed pigs [37]. In the present study, we found a significant downregulation of IL10RB, CXCL9, CXCL10, CCL4, and some other genes (Table 2), which are some of the most powerful inflammatory markers. Stoeker et al [42] revealed that the expression of these genes might entail a risk of making the inflammatory response worse, with lesion formation at tissue stage. Similarly, Pistol et al [22] also investigated the changes in pig liver, which showed a significant reduction in inflammatory cytokine gene expression, as a result of ZEN contamination. The mRNA expression of cytokines was downregulated in the jejunum of DONtreated $(0.9 \mathrm{mg} / \mathrm{kg}$ feed) piglets, and in the ileum of piglets after chronic exposure to low-level DON [43]. We also observed that DON and ZEN exert their effects on the expression of several genes through communication with transcription and signaling factors. These toxins might cause renal failure due to immunosuppression, which might have significant consequences during an infectious process.

A maximum tolerable daily intake for ZEA of $0.5 \mu \mathrm{g} / \mathrm{kg}$ of body weight is now recognized by the Joint Committee $\mathrm{WHO} /$ FAO, based on the no observed-effect level of $40 \mu \mathrm{g} / \mathrm{kg}$ body weight/d found in a 15 day study in pigs. The committee suggested that the total intake for ZEA and its metabolites should not more than this concentration [44]. Prepuberal gilts fed diets polluted with ZEA (0.004 to $0.358 \mathrm{mg} / \mathrm{kg}$ ) exhibited hepatocyte glycogen depletion and expansion of hepatic interlobular connective tissue and hemossiderosis in spleen [45]. In earlier studies, concentrations of DON and ZEN from 1 to $150 \mu \mathrm{M}$ were used alone or in combinations to examine their cooperative or antagonistic effects on inhibition of macromolecular synthesis, DNA methylation and fragmentation. Moreover, the above concentrations are in accordance with the levels possibly encountered in the gastrointestinal tract of human or animals tissues after utilization of food or feed polluted with 2 to $4 \mathrm{mg} / \mathrm{kg}$ of body weight of DON and ZEN [46]. Six pro-inflammatory cytokines of IL1 $\alpha, I L 1 \beta, I L 6$, $I L 8, T N F \alpha$, and monocyte chemotactic genes were up-regulated in the porcine jejunal epithelial cell line in reaction to individual and mixtures of cytotoxic concentrations at DON $2 \mu \mathrm{M}$, and ZEN $40 \mu \mathrm{M}$ treatments. All these pro-inflammatory cytokine shows a significant role in initiation of the inflammatory response in the intestinal epithelial cells [47]. According to Pistol et al [22], 316 parts per billion ZEN contaminated diet reason for desperately reduced the gene expression of pro-inflammatory cytokines, such as TNF- $\alpha, I L-1 \beta, I L-8, I L-6$, and interferon- $\gamma$ in the liver of piglets fed the ZEN contaminated diet. Also observed, DON induced modest expressions of pro-inflammatory genes such as TNF- $\alpha, I L-1 \beta$, and $I L-6$ in the liver relative to the spleen of mice [48]. The mRNA expression of cyclooxygenase- $1, I L-1 \beta$, IL-10, and TLR4 levels were decreased in the jejunum of piglets fed a DON $(0.9 \mathrm{mg} / \mathrm{kg}$ feed) diet when compared to a control diet [49].

Taken together, our results demonstrated that DON- and ZENcontaminated diets induce significant alterations in the global transcriptome levels in pig kidneys. A total of 186 genes with a significantly changed profile compared with the control diet group were found through RNA-seq analyses; among these, 64\% genes were upregulated and 36\% downregulated. Pathway analysis pointed out that DON- and ZEN-affected genes are associated with various diseases and infections. It was also observed that the expression of most of the DEGs involved in inflammatory response was suppressed by DON and ZEN. Based on these results, we postulate that DON and ZEN might have an biphasic effect in vivo, via the suppression of inflammatory response in pigs or any other animal kidneys, which might affect immune homeostasis. This is not only of relevance for a further risk characterization of DON and ZEN exposure in growing pigs and other farm animals, but might also serve as a model for studies trying to estimate interference strategies to alleviate adverse health effects of DON and ZEN.

\section{CONFLICT OF INTEREST}

We certify that there is no conflict of interest with any financial organization regarding the material discussed in the manuscript.

\section{ACKNOWLEDGMENTS}

This work was carried out with the support of "Cooperative Research Program for Agriculture Science and Technology Development (Project No. PJ01093202)" and by the 2016 RDA Fellowship Program of the National Institute of Animal Sciences, Rural Development Administration, Republic of Korea.

\section{REFERENCES}

1.Shephard GS, Thiel PG, Stockenstrom S, Sydenham EW. Worldwide survey of fumonisin contamination of corn and corn-based products. J AOAC Int 1996;79:671-87.

2.Döll S, Dänicke $S$. The Fusarium toxins deoxynivalenol (DON) and zearalenone (ZEN) in animal feeding. Prev Vet Med 2011;102:132-45.

3.Cano PM, Seeboth J, Meurens F, et al. Deoxynivalenol as a new factor in the persistence of intestinal inflammatory diseases: an emerging hypothesis through possible modulation of Th17- mediated response. PLoS One 2013;8:e53647. 
4.Rotter BA, Prelusky DB, Pestka JJ. Toxicology of deoxynivalenol (vomitoxin). J Toxicol Environ Health 1996;48:1-34.

5.Rotter BA, Thompson BK, Clarkin S, Owen TC. Rapid colorimetric bioassay for screening of fusarium mycotoxins. Nat Toxins 1993;1: 303-7.

6.Pestka JJ, Zhou HR, Moon Y, et al. Cellular and molecular mechanisms for immune modulation by deoxynivalenol and other trichothecenes: unraveling a paradox. Toxicol Lett 2004;53:61-73.

7.Diekman MA, Green ML. Mycotoxins and reproduction in domestic livestock. J Anim Sci 1992;70:1615-27.

8. Takemura H, Shim JY, Sayama K, et al. Characterization of the estrogenic activities of zearalenone and zeranol in vivo and in vitro. J Steroid Biochem Mol Biol 2007;103:170-7.

9.Koraichi F, Videmann B, Mazallon M, et al. Zearalenone exposure modulates the expression of $\mathrm{ABC}$ transporters and nuclear receptors in pregnant rats and fetal liver. Toxicol Lett 2012;211:246-56.

10. Ghedira-Chekir L, Maaroufi K, Creppy EE, Bacha H. Cytotoxic and genotoxic effects of zearalenone: prevention by vitamin E. J Toxicol Toxin Rev 1999;18:355-68.

11. National Research Council (NRC). Nutrition requirements of swine. 11th edition. Washington, DC, USA: National Academy Press; 2012.

12. Robinson MD, McCarthy DJ, Smyth GK. edgeR: a Bioconductor package for differential expression analysis of digital gene expression data. Bioinformatics 2010;26:139-40.

13. Reddy KE, Jeong JY, Lee SD, et al. Effect of different early weaning regimens for calves on adipogenic gene expression in Hanwoo loin at the fattening stage. Livest Sci 2017;195:87-98.

14. Dennis G, Jr, Sherman BT, Hosack DA, et al. DAVID: database for annotation, visualization, and integrated discovery. Genome Biol 2003;4:R60.

15. Block SM. Kinesin: what gives? Cell 1998;93:5-8.

16. Do KN, Fink LN, Jensen TE, Gautier L, Parlesak A. TLR2 controls intestinal carcinogen detoxication by CYP1A1. PloS One 2012;7: e32309.

17. Tomlinson IM, Cook GP, Walter G, et al. A complete map of the human immunoglobulin VH locus. Ann NY Acad Sci 1995;764:43-6.

18. Butler JE, Wertz N. Antibody repertoire development in fetal and neonatal piglets. XVII. IgG subclass transcription revisited with emphasis on new IgG3. J Immunol 2006;177:5480-9.

19. Tollin M, Bergman P, Svenberg T, Jornvall H, Gudmundsson GH, Agerberth B. Antimicrobial peptides in the first line defence of human colon mucosa. Peptides 2003;24:523-30.

20. Cheng AL, Huang WG, Chen ZC, et al. Identification of novel nasopharyngeal carcinoma biomarkers by laser capture microdissection and proteomic analysis. Clin Cancer Res 2008;14:435-45.

21. Hikami K, Ehara Y, Hasegawa M, et al. Association of IL-10 receptor 2 (IL10RB) SNP with systemic sclerosis. Biochem Biophys Res Commun 2008;373:403-7.

22. Pistol GC, Gras MA, Marin DE, et al. Natural feed contaminant zearalenone decreases the expressions of important pro- and anti-inflammatory mediators and mitogen-activated protein kinase/NF-kappaB signalling molecules in pigs. Br J Nutr 2014;111:452-64.
23. Dufour JH, Dziejman M, Liu MT, et al. IFN-gamma-inducible protein 10 (IP-10; CXCL10)-deficient mice reveal a role for IP-10 in effector T cell generation and trafficking. J Immunol 2002;168:3195-204.

24. Altara R, Manca M, Hessel MH, et al. CXCL10 is a circulating inflammatory marker in patients with advanced heart failure: a pilot study. J Cardiovasc Transl 2016;9:302-14.

25. Johannes FW, Martani JL, Lissinda HD, Lizelle Z. Evaluation of the cytotoxic properties, gene expression profiles and secondary signalling responses of cultured cells exposed to fumonisin B1, deoxynivalenol and zearalenone mycotoxins. Arch Toxicol 2016;91:2265-82.

26. Shen Q, Singh P. Identification of a novel SP3 binding site in the promoter of human IGFBP4 gene: role of SP3 and AP-1 in regulating promoter activity in CaCo2 cells. Oncogene 2004;23:2454-64.

27. Wan D, Wang X, Wu Q, et al. Integrated transcriptional and proteomic analysis of growth hormone suppression mediated by trichothecene T-2 toxin in rat GH3 cells. Toxicol Sci 2015;147:326.

28. Kalaria RN, Kroon SN. Complement inhibitor C4-binding protein in amyloid deposits containing serum amyloid P in Alzheimer's disease. Biochem Biophys Res Commun 1992;186:461-6.

29. Kaplan R, Morse B, Huebner K, et al. Cloning of three human tyrosine phosphatases reveals a multigene family of receptor-linked proteintyrosine-phosphatases expressed in brain. Proc Natl Acad Sci USA 1990; 87:7000-4.

30. Goyarts T, Dänicke S, Tiemann U, Rothkötter HJ. Effect of the Fusarium toxin deoxynivalenol (DON) on IgA, IgM and IgG concentrations and proliferation of porcine blood lymphocytes. Toxicol In Vitro 2006;20;858-67.

31. Li M, Harkema JR, Cuff CF, Pestka JM. Deoxynivalenol exacerbates viral Bronchopneumonia induced by respiratory reovirus infection. Toxicol Sci 2007;95:412-26.

32. Kim SH, Park YY, Kim SW, et al. ANGPTL4 induction by prostaglandin E2 under hypoxic conditions promotes colorectal cancer progression. Cancer Res 2011;71:7010-20.

33. Amuzie, CJ, Shinozuka J, Pestka JJ. Induction of suppressors of cytokine signaling by the trichothecene deoxynivalenol in the mouse. Toxicol Sci 2009;111:277-87.

34. Croker BA, Kiu H, Nicholson SE. SOCS regulation of the JAK/STAT signalling pathway. Semin Cell Dev Biol 2008;19:414-22.

35. Wu Q, Dohnal V, Huang L, Kuca K, Yuan Z. Metabolic pathways of trichothecenes. Drug Metab Rev 2010;42:250-67.

36. Gu MJ, Song SK, Lee IK, et al. Barrier protection via Toll-like receptor 2 signaling in porcine intestinal epithelial cells damaged by deoxynivalnol. Vet Res 2016;47:25.

37. Pistol GC, Braicu C, Motiu M, et al. Zearalenone mycotoxin affects immune mediators, MAPK Signalling molecules, nuclear receptors and genome-wide gene expression in pig spleen. PLoS ONE 2015; 10:e0127503.

38. Hueza IM, Tanabe VK, Benassi JC, Raspantini LER, Gorniak SL. Zearalenone: An estogenic mycotoxin with immunotoxic effects. 8th International Symposium on Poisonous Plants; 2009 May; Paraiba, Brazil.

39. Bolognesi C. Genotoxicity of pesticides: A review of human biomoni- 
toring studies. Mutat Res 2003;543:251-72.

40. Zhang ZQ, Wang SB, Wang RG, et al. Phosphoproteome analysis reveals the molecular mechanisms underlying deoxynivalenolinduced intestinal toxicity in IPEC-J2 cells. Toxins 2016;8:270.

41. Liang Z, Ren Z, Gao S, et al. Individual and combined effects of deoxynivalenol and zearalenone on mouse kidney. Environ Toxicol Pharmacol 2015;40:686-91.

42. Stoeker L, Nordone S, Gunderson S, et al. Assessment of Lactobacillus gasseri as a candidate oral vaccine vector. Clin Vaccine Immunol 2011;18:1834-44.

43. Becker C, Reiter M, Pfaffl MW, et al. Expression of immune relevant genes in pigs under the influence of low doses of deoxynivalenol (DON). Mycotoxin Res 2011;27:287-93.

44. CCFAC. Codex Committee on Food Additives and Contaminants. Posting date. Joint FAO/WHO Expert Committee on Food Additives: Position paper on zearalenone. Publication CCFAC 00/19. Rome, Italy: Codex Alimentarius Commission; 2000.

45. Tiemann U, Brüssow KP, Jonas L, et al. Effects of diets with cereal grains contaminated by graded levels of two Fusarium toxins on selected immunological and histological parameters of spleen in gilts. J Anim Sci 2006;84:236-45.

46. Kouadio JH, Mobio TA, Baudrimont I, et al. Comparative study of cytotoxicity and oxidative stress induced by deoxynivalenol, zearalenone or fumonisin B1 in human intestinal cell line Caco-2. Toxicology 2005;213:56-65.

47. Jung HC, Eckmann L, Yang SK, et al. A distinct array of proinflammatory cytokines is expressed in human colon epithelial cells in response to bacterial invasion. J Clin Invest 1995;95:55-65.

48. Pestka JJ, Amuzie CJ. Tissue distribution and proinflammatory cytokine gene expression following acute oral exposure to deoxynivalenol: comparison of weanling and adult mice. Food Chem Toxicol 2008;46: 2826-31.

49. Alizadeh A, Braber S, Akbari P, Garssen J, Gremmels JF. Deoxynivalenol impairs weight gain and affects markers of gut health after low-dose, short-term exposure of growing pigs. Toxins 2015;7:2071-95. 